\title{
Synthese und Eigenschaften von 3-Äthoxy-1.2-dithiolium-Salzen
}

\author{
J. FAUST und J. FABIAN \\ Institut für Organische Chemie der Technischen Universität Dresden \\ (Z. Naturforschg. 24 b, 577-580 [1969] ; eingegangen am 23. Oktober 1968)
}

\begin{abstract}
Die farblosen, feuchtigkeitsempfindlichen 3-Äthoxy-1.2-dithioliumfluoborate $\left(1, \mathrm{X}=\mathrm{BF}_{4}\right)$ können durch Umsetzung von 1.2-Dithiolonen-(3) (2) mit Triäthyloxoniumfluoborat in Dichlormethan erhalten werden. Das Fluoborat-Anion ist austauschbar, ein nucleophiler Angriff ist an verschiedenen Stellen des reaktionsfähigen Moleküls möglich. Dialkylsulfate, Alkyltosylate und Alkylhalogenide sind zur Alkylierung der 2 ungeeignet, die Umsetzung von 3-Chlor-1.2-dithiolium-Salzen (4) mit Alkoholen führt ebenfalls nicht zu 3-Alkoxy-1.2-dithiolium-Verbindungen.

Die charakteristischen Absorptionen der 3-Äthoxy-1.2-dithioliumionen (1) im Ultravioletten werden angegeben und durch SCF-LCI-PPP-Berechnungen theoretisch abgeleitet. Ein Vergleich dieser Absorptionen mit den Spektren der 1.2-Dithiolone-(3) (2) in konzentrierter Schwefelsäure zeigt, daß die Carbonyl-Verbindungen unter diesen Bedingungen am Sauerstoff protoniert werden.
\end{abstract}

Die Alkylierung eines 1.2-Dithiolons-(3) (2) ist bisher nicht gelungen. Methyljodid ${ }^{1}$, Benzylbromid, p-Bromphenacylbromid und Methyltosylat wirken auf 2 nicht ein. Auch Dimethylsulfat ${ }^{1}$, mit dem die iso- $\pi$-elektronischen 4 H-Thiopyranone-(4) glatt in 4-Methoxy-thiopyryliummethosulfate überführt werden können ${ }^{2}$, sowie Diäthylsulfat, reagieren mit 2 selbst unter schärferen Bedingungen nicht.

Die Synthese von 3-Methoxy-1.2-dithioliumsalzen (5) oder 3-Äthoxy-1.2-dithioliumsalzen (1) gelingt auch nicht durch Umsetzung geeigneter 3-substituierter 1.2-Dithioliumsalze mit Methanol oder Äthanol. 3-Chlor-1.2-dithioliumchloride (4) ${ }^{3}$ reagieren mit 2 Molen Alkohol zum 1.2-Dithiolon-(3) (2), daneben entsteht in der Regel auch 1.2-Dithiolthion-(3) (3) ${ }^{4}$. Äthanol wird dabei zu Diäthyläther alkyliert. Bringt man molare Mengen 4 und Äthanol in Acetonitril zur Reaktion, läßt sich nach vollständiger Umsetzung des Alkohols noch reichlich 4 nachweisen. Offenbar reagiert das gebildete 3-Äthoxy-1.2-dithioliumsalz (1) rascher mit dem Äthanol als das 3Chlor-1.2-dithioliumchlorid (4) ${ }^{5}$.

Es gelang uns nun, durch Umsetzung von 1.2-Dithiolon-(3) (2) mit Triäthyloxoniumfluoborat ${ }^{6}$ in Dichlormethan 3-Äthoxy-1.2-dithioliumfluoborate (1, $\mathrm{X}=\mathrm{BF}_{4}$ ) zu erhalten. Mit Ausnahme des Esters $2 \mathrm{~d}$ reagierten alle eingesetzten $\mathbf{2}$ in teilweise sehr hohen Ausbeuten.

3-Äthoxy-1.2-dithioliumfluoborate $\left(\mathbf{1}, \mathrm{X}=\mathrm{BF}_{4}\right)$ stellen gut kristallisierte Verbindungen dar, die be-

1 H. Prinzbach u. E. Futterer, in: Advances in Heterocyclic Chemistry, Bd. 7, 53, Herausgeber A. R. KATritzKY u. A. J. Boulton, Academic Press, New York und London 1966

2 G. Traverso, Ann. Chimica 47, 1244 [1957].

3 J. Faust u. R. Mayer, Liebigs Ann. Chem. 688, 150 [1965].

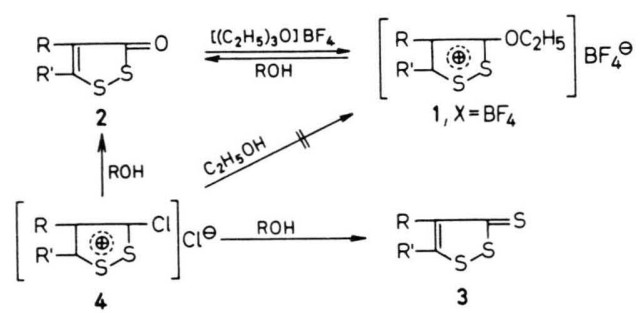

$\mathrm{a}: \mathrm{R}=\mathrm{H}, \mathrm{R}^{\prime}=\mathrm{H} ; \mathrm{b}: \mathrm{R}=\mathrm{C}_{2} \mathrm{H}_{5}, \mathrm{R}^{\prime}=\mathrm{H} ; \mathrm{c}: \mathrm{R}-\mathrm{R}^{\prime}=\left(\mathrm{CH}_{2}\right)_{4}$; d: $\mathrm{R}=\mathrm{H}, \mathrm{R}^{\prime}=\mathrm{COOC}_{2} \mathrm{H}_{5}$; e: $\mathrm{R}=\mathrm{C}_{6} \mathrm{H}_{5}, \mathrm{R}^{\prime}=\mathrm{H} ; \mathrm{f}: \mathrm{R}=\mathrm{H}$, $\mathrm{R}^{\prime}=\mathrm{C}_{6} \mathrm{H}_{5} ; \mathrm{g}: \mathrm{R}-\mathrm{R}^{\prime}=\mathrm{CH}=\mathrm{CH}-\mathrm{CH}=\mathrm{CH}$.

reits gegen Luftfeuchtigkeit sehr empfindlich sind und beim Erwärmen mit niederen Alkoholen 2 zurückbilden. Die Reaktion mit Basen folgt jedoch keinem einheitlichen Schema. Es tritt in Abhängigkeit von der Art des Nucleophils und den Reaktionsbedingungen Ringöffnung, Substitution in 3-Stellung, Eliminierung der Äthylgruppe oder Austausch des Ringschwefels ein ${ }^{5}$. Diese Reaktionsfähigkeit läßt sich bereits an Hand eines statischen Reaktivitätsmodells, das von den berechneten Moleküldiagrammen (vgl. z. B. Abb. 1) ausgeht, zwanglos erklären.

Der Austausch des Fluoborat-Anions ist erwartungsgemäß nur in absolut wasserfreiem Medium möglich. Mit Perchlorsäure in Acetanhydrid erhält man farblose, kristalline, zersetzliche Verbindungen $\left(1, \mathrm{X}=\mathrm{ClO}_{4}\right)$, deren spektrale Eigenschaften mit denen der $1, \mathrm{X}=\mathrm{BF}_{4}$ völlig übereinstimmen.

4 Vgl. dazu J. Faust, H. Spies u. R. Mayer, Z. Chem. 7, 275 [1967].

5 Veröffentlichung in Vorbereitung.

6 H. Meerwein, in: Houben-Weyl, Methoden der Organischen Chemie, Bd. VI/3, Georg Thieme, Stuttgart 1965, S. 336 . 


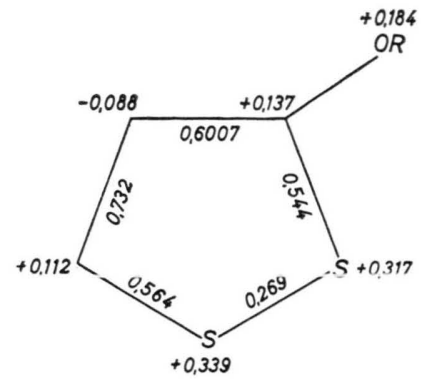

Abb. 1. Moleküldiagramm des 3-Methoxy-1.2-dithioliumions (1 a) nach einer SCF - PPP-Berechnung.

Diäthoxycarboniumhexachloroantimonat ${ }^{7}$ setzt sich ebenfalls mit $\mathbf{2}$ um, es konnte jedoch bisher kein analysenreines 3-Äthoxy-1.2-dithioliumhexachloroantimonat $\left(1, \mathrm{X}=\mathrm{SbCl}_{6}\right)$ erhalten werden. Reaktionen mit Basen deuten darauf hin, daß auch eine $O$. Alkylierung stattgefunden hat. 1.2-Dithiolthione-(3) (3) können sowohl mit Triäthyloxoniumfluoborat als auch mit Diäthoxycarboniumhexachloroantimonat glatt zu 3-Äthylmercapto-1.2-dithioliumsalzen (1, $\mathrm{SC}_{2} \mathrm{H}_{5}$ statt $\mathrm{OC}_{2} \mathrm{H}_{5}$ ) umgesetzt werden.

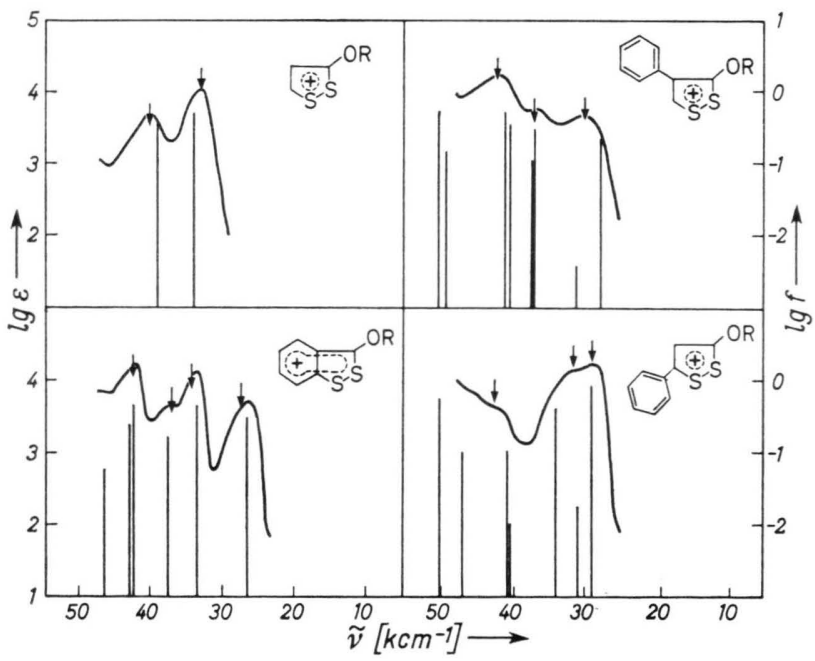

Abb. 2. Spektrale Absorptionen der 3-Hydroxy-1.2-dithioliumionen (6) (Kurve), der 3-Äthoxy-1.2-dithioliumionen (1) (Maxima und Schultern als Pfeile) und berechnete Ubergänge der 3-Methoxy-1.2-dithioliumionen (5) (als senkrechte Linien; Parameter: l. c. ${ }^{11,12}$ und exocyclische $\beta_{\mathrm{CC}}=$ $-1,74 \mathrm{eV})$.

7 H. Meerwein, K. Bodenbrenner, P. Borner, F. Kunert u. K. Wunderlich, Liebigs Ann. Chem. 632, 38 [1960] ; S. Kabuss, Angew. Chem. 78, 714 [1966].

8 R. Zahradník, in: Physical Methods in Heterocyclic Chemistry 5, 150, Herausgeber: A. R. Katritzky, Academic Press, New York und London 1965.
3-Äthoxy-1.2-dithioliumionen (1) sind in konzentrierter Schwefelsäure ausreichend beständig, um die Absorption im Ultravioletten zu bestimmen. Die charakteristischen Absorptionen des 3- Äthoxy-1.2dithioliumions (1 a), des 3-Äthoxy-4-phenyl-1.2-dithioliumions (1 e), des 3-Äthoxy-5-phenyl-1.2-dithioliumions (1 f) und $\operatorname{des} 3$ Äthoxy benzo $1.2 \mathrm{di}$ thioliumions ( $\mathbf{l} \mathbf{g})$ sind in Abb. 2 wiedergegeben. Sie unterscheiden sich auffallend stark und deuten auf keine gemeinsame Absorptionseigenschaft des heterocyclischen Fünfringes hin. Ein Vergleich von Spektraldaten der nichtäthoxysubstituierten Verbindungen ${ }^{8}$ läßt auch ein solches Verhalten nicht erwarten.

Ein Verständnis der spektralen Unterschiede von $\mathbf{1 a}, \mathbf{1 e}, \mathbf{1} \mathbf{f}$ und $\mathbf{1} \mathrm{g}$ ist durch halbempirische SCF-LCI-Berechnungen in der von PARISER, PARR und POPLE angegebenen Näherung möglich. Überraschend gute Ergebnisse werden auch ohne Berücksichtigung der Ladung in Form einer VESCF-Prozedur erhalten ${ }^{9}$. Die $\pi-\pi^{*}$-Übergänge, die unter Beibehalt der früher verwendeten Näherung ${ }^{10}$ und Heteroatomparameter ${ }^{11,12}$ berechnet wurden, sind in Abb. 2 eingezeichnet. Nicht nur die Absorptionsbereiche, sondern auch die relativen Intensitäten (die Logarithmen der Oszillatorenstärke $f$ sind näherungsweise den Logarithmen der Extinktionskoeffizienten $\varepsilon$ proportional) werden richtig wiedergegeben. Die Berechnungen unterstreichen überzeugend den unterschiedlichen Einfluß der Phenylsub. stitution.

Die Kenntnis der spektralen Absorptionen der 3-Äthoxy-1.2-dithioliumionen (1) ist auch geeignet, das Verhalten der 1.2-Dithiolone-(3) (2) in konzentrierter Schwefelsäure zu klären. Da die Spektren von 2 in diesem Lösungsmittel weitgehend den Spektren von $\mathbf{1}$ entsprechen (Abb. 2), sollten die $\mathbf{2}$ unter diesen Bedingungen als 3-Hydroxy-1.2-dithioliumionen (6) vorliegen. Konzentrierte Schwefelsäure führt also zur O-Protonierung der 1.2-Dithiolone(3) (2) :

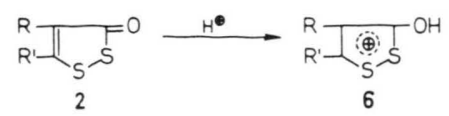

9 J. Fabian, A. Mehlhorn u. R. Zahradník, Theoret. chim. Acta 12, 247 [1968].

10 J. Fabian, A. Mehlhorn u. R. Zahradník, J. physic. Chem. 72, 3975 [1968].

11 J. Fabian, K. Fabian u. H. Hartmann, Theor. chim. Acta 12, 319 [1968].

12 H. Hartmann u. J. Fabian, Ber. Bunsenges., im Druck. 


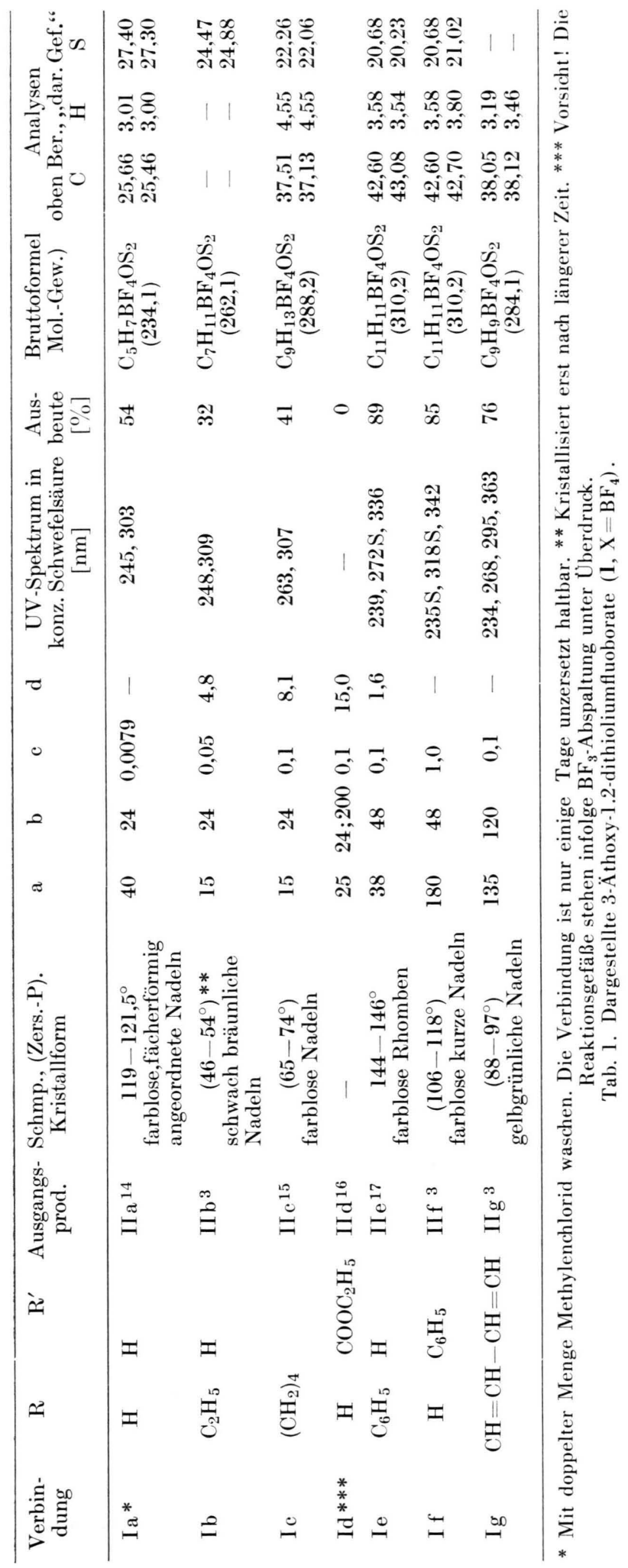

\section{Experimenteller Teil}

\section{3-Athoxy-1.2-dithioliumfluoborate $\left(1, X=B F_{4}\right)$}

Allgemeine Arbeitsvorschrift: Man löst 0,1 Mol 1.2-Dithiolon-(3) (2) in $\alpha \mathrm{ml}$ wasserfreiem Methylenchlorid und fügt 19,0 g $(0,1 \mathrm{Mol})$ frisch dargestelltes Triäthyloxoniumfluoborat ${ }^{6}$ in $40 \mathrm{ml}$ wasserfreiem Methylenchlorid zu. Nach $b$ Stdn. wird das Lösungsmittel bei 25 bis $35^{\circ}$ Badtemperatur im Vakuum entfernt, der größtenteils kristalline Rückstand in einer Glasfritte mit $6 \mathrm{ml}$ wasserfreiem Methylenchlorid durchfeuchtet und sofort scharf abgesaugt. Man wäscht rasch mit einem Gemisch von $50 \mathrm{ml}$ Methylenchlorid und $50 \mathrm{ml}$ Diäthyläther (wasser- und peroxidfrei), darauf noch zweimal mit je $100 \mathrm{ml}$ Diäthyläther. Die so erhaltenen Salze sind höchstens noch schwach gefärbt, bereits recht rein und wurden für alle weiteren Reaktionen direkt eingesetzt. Die angegebenen Ausbeuten sind auf dieses Produkt bei einem c-molaren Ansatz berechnet. Die Umkristallisation aus Acetanhydrid führt zu analysenreinen Substanzen, ist aber in der Regel sehr verlustreich.

Ein Teil des Ausgangsprodukts 2 kann wiedergewonnen werden, indem man sämtliche Filtrate vereinigt, mit $5 \mathrm{ml}$ Äthanol erwärmt, mit Wasser wäscht und trocknet. Nach Abdestillieren des Lösungsmittels werden aus dem Rückstand durch Kristallisation oder Destillation $d$ g 1.2-Dithiolon-(3) (2) gewonnen. Beispiele in Tab. 1.

\section{3-Äthoxy-5-phenyl-1.2-dithioliumperchlorat (l f , $\mathrm{X}=\mathrm{ClO}_{4}$ )}

$0,93 \mathrm{~g}(3 \mathrm{mMol})$ feinverteiltes 3-Äthoxy-5-phenyl1.2-dithioliumfluoborat $\left(\mathbf{1} \mathbf{f}, \mathrm{X}=\mathrm{BF}_{4}\right)$ werden in $5 \mathrm{ml}$ gereinigtem Acetanhydrid suspendiert und unter Umrühren mit 0,8 g 70-proz. Perchlorsäure versetzt. Unter starker Erwärmung entsteht eine homogene, bräunliche Lösung, aus der nach einigen Min. ein farbloses Kristallisat ausfällt. (Vorsicht! Die Reaktion kann explosionsartig verlaufen!) Man wäscht das erhaltene Perchlorat zweimal mit je $0,5 \mathrm{ml}$ Acetanhydrid, anschließend mit reichlich wasser- und peroxidfreiem Diäthyläther.

0,46 g (48\% d. Th.) farblose, kurze Nadeln (Zersetzungspunkt $81-113^{\circ}$ ), die nach einigen Monaten gelb und schmierig geworden sind.

$$
\begin{array}{rrrr}
\mathrm{C}_{11} \mathrm{H}_{11} \mathrm{ClO}_{5} \mathrm{~S}_{2} & (322,8) \\
\text { Ber. } & \text { Cl } 10,98 & \text { S } & 19,87, \\
\text { Gef. } & \text { Cl } 11,37 & \text { S } & 19,84 .
\end{array}
$$

13 R. Mayer, P. Wittig, J. Fabian u. R. Heitmüller, Chem. Ber. 97, 654 [1964].

14 R. Mayer u. J. Faust, Chem. Ber. 96, 2702 [1963].

15 P. Raoul u. J. Vialle, Bull. Soc. chim. France 1959, 1670.

16 E. Klingsberg, Chem. and Ind. 1960, 1568.

17 R. F. C. Brown, I. D. Rae u. S. Sternhell, Austral. J. Chem. 18, 1211 [1965]. 


\section{3-Äthylmercapto-4-phenyl-1.2-dithioliumfluoborat $\left(\mathbf{1}\right.$ e, $\mathrm{SC}_{2} \mathrm{H}_{5}$ statt $\mathrm{OC}_{2} \mathrm{H}_{5} ; \mathrm{X}=\mathrm{BF}_{4}$ )}

Man löst 10,5 g (50 mMol) 4-Phenyl-1.2-dithiolthion- (3) (3 e) ${ }^{13}$ in $125 \mathrm{ml}$ und $9,5 \mathrm{~g}(50 \mathrm{mMol})$ Triäthyloxoniumfluoborat ${ }^{6}$ in $20 \mathrm{ml}$ wasserfreiem Methylenchlorid und vereinigt die Lösungen. Einige Stdn. später beginnt die rote Farbe der Lösung merklich zu verblassen, nach 12 Tagen ist die Hauptmenge des Fluoborats auskristallisiert. Nach Waschen mit wenig absolutem Äthanol 11,8 g (73\% d. Th.) große, gelbgrüne Säulen vom Schmp. $145-148^{\circ}$ (Zersetzung).

$\mathrm{C}_{11} \mathrm{H}_{11} \mathrm{BF}_{4} \mathrm{~S}_{3} \quad(326,2)$

Ber. C 40,50 H 3,40 ,

Gef. C 40,44 H 3,18 .

Einfluß von Effektoren auf Molekulargewicht und Aktivität der GlutamatDehydrogenase $(\mathrm{GluDH})$ aus Rinderleber in vitro

\title{
I. Das Molekulargewicht der GluDH und ihrer Untereinheiten
}

\author{
K.-O. Mosebach und M. Kempfle
}

Physikalisch-chemische Abteilung des physiologisch-chemischen Instituts der Universität Bonn

(Z. Naturforschg. 24 b, 580-582 [1969] ; eingegangen am 28. November 1968)

\begin{abstract}
We studied the molecular weight of cristalline GluDH from beef liver 1) in different dilutions, 2) after treatment with NADH and diethylstilbestrol, 3) after treatment with $6 \mathrm{~m}$ guanidine-HCl. When we diluted GluDH there was a spontaneous dissociation of the molecule into subunits. The molecular weight decreased from $2,000,000$ (at $20 \mathrm{mg} / \mathrm{ml}$ ) to 310,000 (at $0.01 \mathrm{mg} / \mathrm{ml}$ ). After concentrating the solution again we got back the associated molecule. After treatment with NADH and diethylstilbestrol we obtained a dissociation of GluDH into subunits, too, but with a significant loss of enzymatic activity. This effect is reversibel - after dialysis we found the associated molecule again. When we treated $\mathrm{GluDH}$ with $6 \mathrm{~m}$ guanidine- $\mathrm{HCl}$ we found a complete dissociation of the molecule into subunits with a molecular weight of 52,000 . This treatment caused an irreversibel loss of enzymatic activity.
\end{abstract}

Bei der Aufklärung des chemischen Wirkungsmechanismus von Hormonen besteht die Notwendigkeit, mögliche Einzelreaktionen, die mit Sicherheit nur Teil eines komplexen Geschehens sind, losgelöst vom lebenden Organismus zu studieren. $\mathrm{Zu}$ solchen möglichen Reaktionen gehört der Einfluß von Hormonen auf die Konformation von Protein-, insbesondere Enzymmolekülen. Beim Durchdenken der bestehenden Theorien über die Wirkungsweise von Hormonen stößt man zum Schluß immer wieder auf die Vorstellung einer allosterischen Wirkung auf die hochpolymeren Substanzen der Zelle. YIELDING und Tomkins ${ }^{1}$ gelang vor Jahren der Nachweis, daß eine Reihe von Steroidhormonen und anderen Reagenzien das Mol.-Gew. der GluDH verändern kann. Von besonderem Interesse ist hierbei der gleichzeitige Einfluß auf die Enzymaktivität. Eine Anzahl von Fragen ist ungeklärt geblieben, die es zweckmäßig er-

1 L. K. Yielding u. G. M. Tomkins, Recent progress in Hormone Res. 18, 467 [1962] und dortige Literatur.

2 K.-O. Mosebach, H. Jühe u. W. Dirscherl, Acta Endocr. [Kobenhavn] 54, 557 [1967]; K.-O. Mosebach, in: „13. Sympos. der deutschen Endokrinologischen Gesellschaft", Springer-Verlag, Berlin-Heidelberg - New York 1968 , p. 17; U. Lippert, K.-O. Mosebach u. G. KramPITZ, Nature [London] 214, 917 [1967] ; K.-O. MOSEBACH, scheinen ließen, sich im Rahmen unserer Forschungen über die Wirkungsweise von Steroidhormonen ${ }^{2}$ mit diesem Problem nochmals auseinanderzusetzen.

$\mathrm{Zu}$ den nicht einwandfrei geklärten Fragen gehört bereits die nach dem Mol.-Gew. der GluDH. Auf die Bestimmung des Mol.-Gew. dieses Enzyms wird in dieser Arbeit eingegangen. Enzymatisch aktive GluDH zerfällt beim Verdünnen von hohen zu niedrigen Konzentrationen in kleinere, ebenfalls enzymatisch aktive Einheiten. Dieser Prozeß beginnt bei etwa $5 \mathrm{mg} / \mathrm{ml}$ und wurde von uns bis $0,01 \mathrm{mg} / \mathrm{ml}$ verfolgt. Das Mol.-Gew. der assoziierten Form beträgt etwa 2000000 . Für das Mol.-Gew. der Untereinheiten sind verschiedene Werte angegeben worden. FrIEDEN ${ }^{3}$ ermittelte 400000 , das entspricht 5 Untereinheiten; YIELding und TOMKINS ${ }^{1}$, SUND ${ }^{4}$ sowie BAYley und RADDA ${ }^{5}$ fanden 250000 , entsprechend 8 Untereinheiten. Der von uns ${ }^{2 a}$ mit Hilfe

W. Dirscherl, K. Rath u. G. Rieck, Hoppe Seyler's Z. physiol. Chem. 344, 197 [1966].

2a M. Kempfle, K.-O. Mosebach u. W. Dirscherl, 5. FEBSMeeting, Prague 1968, Abstr.No. 1090.

3 C. Frieden, J. biol. Chemistry 242. 1705 [1967].

${ }^{4}$ H. Sund, Acta chem. scand. 17, 1502 [1963].

5 P. A. BAyley u. G. K. RadDA, Biochem. J. 98, 105 [1966]. 